\title{
ASSORTIVE MATING IN A POPULATION IN WHICH TWO ALLELES ARE SEGREGATING
}

\author{
P. O'DONALD \\ Department of Genetics, University of Cambridge
}

Received 3.viii.6o

\section{INTRODUCTION}

That positive assortive mating, the tendency of like to mate with like, occurs in man has long been established. The phenomenon was studied by Fisher (I9I8). More recently, assortive mating has been found in several polymorphic wild populations. Cooch and Beardmore (I959) found assortive mating in the Blue-Snow Goose complex, and O'Donald (1959) has observed assortive mating among the pale and intermediate and dark phases of the Arctic Skua. In this paper, systems involving assortive mating in populations in which two alleles are segregating will be considered.

\section{THE ORIGIN OF ASSORTIVE MATING}

The Arctic Skua is of particular interest here as it shows a cline in its phases: the pale phase predominates in the northern part of the range, and dark and intermediate phases predominate in the south. Fisher (1930) has discussed the evolution of such species, where certain alleles are at an advantage at one end of the range, and pass through an intermediate region of diminishing, neutral and negative advantage to the other end of the range. A genetic equilibrium will be established, Fisher suggests " if the increase in frequency in the favourable region and the decrease in frequency in the unfavourable region, not only balance each other quantitatively, but are equal to the rate at which genes diffuse by migration and sexual union, from the one region to the other ... The longer such an equilibrium is maintained the more numerous will the genetic differences between the types inhabiting extreme regions tend to become, for the situation allows of the extinction of neither the gene favoured locally nor its allelomorph favoured elsewhere, and all new mutations appearing in the intermediate zone which are advantageous at one extreme but disadvantageous at the other will have a chance of being added to the factors in which they differ." So far, there has been no loss of unity in the ancestry of the species. Congenital variations in the means of dispersal will, however, as Fisher points out, lead to the elimination of those individuals that diffuse most, and thus to an increase in genetic isolation. Any tendency among some of the members of the species to assortive mating will still further increase the isolation of the two groups and so diminish the diffusion of the alien gene-complexes in that group where they will be 
eliminated. Any genetic incompatibility that arises will further increase this selective effect.

Clines in nature provide a fairly common example of one type of disruptive selection; in general, disruptive selection of a quantitative character controlled at a number of different loci should also give rise, under certain conditions, to assortive mating. Fisher (1930) says of assortive mating in man: "Its principal biometric effects seem to be to increase the genetic variance produced by a given number of Mendelian factors with given gene ratios, and so to increase in a fixed proportion the intensity of the selection to which each is exposed." Thus if disruptive selection is favouring the more extreme deviants in a population while individuals near the mean are at a disadvantage, then those individuals mating with like mates will contribute a greater proportion of descendants to future generations than those mating with dissimilar partners whose offspring, tending to be more heterozygous, will have characters nearer the population mean. Individuals with an inherited preference towards assortive mating will thus increase in the population.

Mather (1955) has argued that stable polymorphism can be the outcome of disruptive selection. This will be so "whenever we can discern a sufficiently strong tie, or co-operation, between structures or entities, whether of genetic structures within the nucleus, of cellular structures within the zygote, or of zygotes themselves within the population, we might expect to find all the features of polymorphism, viz., sharply delimited classes of the structure or entity adjusted to work together for their common advantage, the adjustment depending on a common genotype which can ensure the necessary conditions, and on a switching system whether environmental, segregational or cytoplasmic to direct the structures into one path or the other." Under a system of this kind, it is clear that assortive mating cannot evolve since positive assortive mating implies independence of the optimal phenotypes, one of Mather's conditions for isolation. Similarly in the disruptive selection of a cline, a tie sufficiently strong to inhibit the evolution of assortive mating might result between the alleles, if, under certain conditions, the heterozygote were at an advantage. This system might, however, lead to a balanced equilibrium between the heterozygous advantage and the selection to limit diffusion and thus to a stable polymorphic cline.

\section{ASSORTIVE MATING WHEN ONE ALLELE IS DOMINANT}

It will be assumed that a proportion a mate with like phenotypes while $\mathrm{I}-\alpha$ mate at random. Suppose in a population comprised of the genotypes $\mathrm{AA}, \mathrm{AB}$ and $\mathrm{BB}$ in the proportion $u, v$ and $w, \mathrm{~A}$ is dominant, so that $\mathrm{AA}$ and $\mathrm{AB}$ are not recognisably distinct. Let $\alpha(u+v)$ of $\mathrm{A}$ phenotypes and aw of $\mathrm{B}$ phenotypes mate with like phenotypes while the remainder of the population mates at random. Then among the A phenotypes the tendency to assortive mating will 
yield the matings $\mathrm{AA} \times \mathrm{AA}, \mathrm{AA} \times \mathrm{AB}$ and $\mathrm{AB} \times \mathrm{AB}$ in the proportions $\frac{u^{2}}{(u+v)^{2}}, \frac{2 u v}{(u+v)^{2}}$ and $\frac{v^{2}}{(u+v)^{2}}$ out of a total of $\alpha(u+v)$. Thus we shall have

Mating type

Frequency

$\begin{array}{ll}\mathrm{AA} \times \mathrm{AA} & a u^{2} /(u+v)+(\mathrm{I}-\alpha) u^{2} \\ \mathrm{AA} \times \mathrm{AB} & 2 a u v /(u+v)+2(\mathrm{I}-\alpha) u v \\ \mathrm{AA} \times \mathrm{BB} & 2(\mathrm{I}-\alpha) u w \\ \mathrm{AB} \times \mathrm{AB} & a v^{2} /(u+v)+(\mathrm{I}-\alpha) v^{2} \\ \mathrm{AB} \times \mathrm{BB} & 2(\mathrm{I}-\alpha) v w \\ \mathrm{BB} \times \mathrm{BB} & \alpha w+(\mathrm{I}-\alpha) w^{2}\end{array}$

Whence

$$
\begin{aligned}
u^{\prime} & =\alpha\left(u+\frac{1}{2} v\right)^{2} /(u+v)+(\mathrm{I}-\alpha)\left(u+\frac{1}{2} v\right)^{2} \\
v^{\prime} & =\alpha v\left(u+\frac{1}{2} v\right) /(u+v)+2(\mathrm{I}-\alpha)\left(u+\frac{1}{2} v\right)\left(w+\frac{1}{2} v\right) \\
w^{\prime} & =\alpha w+\alpha v^{2} / 4(u+v)+(\mathrm{I}-\alpha)\left(w+\frac{1}{2} v\right)^{2}
\end{aligned}
$$

where $u^{\prime}, v^{\prime}$, and $w^{\prime}$ represent the genotypic proportions in the following generation. If we let $p$ be the frequency of the $\mathrm{A}$ allele and $q$ the frequency of $B$, we have

$$
\begin{aligned}
p^{\prime} & =u^{\prime}+\frac{1}{2} v^{\prime}=\left(\alpha p^{2}+\alpha v p / 2\right) /(u+v)+(\mathrm{I}-\alpha) p \\
& =\alpha p\left(p+\frac{1}{2} v\right) /\left(p+\frac{1}{2} v\right)+(\mathrm{I}-\alpha) p \\
& =p .
\end{aligned}
$$

Thus the gene frequency remains constant. We may therefore put $u=p-\frac{1}{2} v$ and $w=q-\frac{1}{2} v$ to obtain a recurrence relation in the variable $v$ alone:

$$
v^{\prime}=\alpha v p /\left(p+\frac{1}{2} v\right)+2(\mathrm{I}-\alpha) p q .
$$

The equilibrium position. At equilibrium, when the population is undergoing no further change, we shall have $v^{\prime}=v$. Thus

$$
v=\alpha v p /\left(p+\frac{1}{2} v\right)+2(\mathrm{I}-\alpha) p q,
$$

which after rearrangement becomes

and

$$
v^{2}+2 p^{2}(\mathrm{I}-\alpha) v-4 p^{2} q(\mathrm{I}-\alpha)=0,
$$

$$
v_{e}=p^{2}(\alpha-\mathrm{I}) \pm p \sqrt{(\mathrm{I}-\alpha)\left[p^{2}(\mathrm{I}-\alpha)+4 q\right]},
$$

where $v_{e}$ denotes the equilibrium value. Clearly for $\alpha<\mathrm{I}$ we must have

$$
v_{e}=p^{2}(\alpha-\mathrm{I})+p \sqrt{(\mathrm{I}-\alpha)\left[p^{2}(\overline{\mathrm{I}}-\alpha)+4 q\right]},
$$

for the alternative root, which we shall call $v_{e}^{*}$, becomes negative. Thus in the case $p=q=\frac{1}{2}$

$$
v_{e}=\frac{1}{4}(\alpha-\mathrm{I})+\frac{1}{4} \sqrt{(\mathrm{I}-\alpha)(9-\alpha)} .
$$

2B 2 
Rate of approach to equilibrium. The recurrence relation in $v$,

$$
v^{\prime}=\alpha v p /\left(p+\frac{1}{2} v\right)+2(\mathrm{r}-\alpha) p q,
$$

is non-linear. However, when rewritten in the form

and by letting $v=y / x$,

$$
v^{\prime}=\frac{2 p v(q+a p)+4(\mathrm{r}-a) p^{2} q}{v+2 p},
$$

we have

Thus

$$
y^{\prime} \mid x^{\prime}=\frac{2 p y(q+a p)+4(\mathrm{r}-a) x p^{2} q}{y+2 p x} .
$$

$$
\left(\begin{array}{l}
y^{\prime} \\
x^{\prime}
\end{array}\right)=\left(\begin{array}{cc}
2 p(q+a p) & 4(\mathrm{I}-a) p^{2} q \\
\mathrm{I} & 2 p
\end{array}\right) \cdot\left(\begin{array}{l}
y \\
x
\end{array}\right)
$$

and we are required to find the $n$th power of the matrix.

The characteristic equation is

or

$$
[2 p(q+\alpha p)-\lambda][2 p-\lambda]-4(\mathrm{r}-\alpha) p^{2} q=0,
$$

Thus

$$
\lambda^{2}-2 p \lambda(\mathrm{r}+q+a p)+4 p^{2} \alpha=0 .
$$

$$
\lambda=p(\mathrm{r}+q+a p) \pm p \sqrt{(\mathrm{r}+q+\alpha p)^{2}-4 \alpha}
$$

which after rearrangement becomes

But

$$
\lambda=p(\mathrm{I}+q+a p) \pm p \sqrt{(\mathrm{I}-\alpha)\left[p^{2}(\mathrm{I}-\alpha)+4 q\right]} .
$$

and

$$
p \sqrt{(\mathrm{r}-a)\left[p^{2}(\mathrm{r}-\alpha)+4 q\right]}=v_{e}-p^{2}(\alpha-\mathrm{r})
$$

Thus

$$
-p \sqrt{(\mathrm{r}-\alpha)\left[p^{2}(\mathrm{r}-\alpha)+4 q\right]}=v_{e}^{*}-p^{2}(\alpha-\mathrm{r}) .
$$

$$
\lambda=v_{e}+2 p \text { or } v_{e}^{*}+2 p .
$$

To find the principal components of frequency we read the columns of the matrix as homogeneous linear functions of the coefficients $a$ and $b$ (Fisher, r949). Thus for the latent root $v_{e}+2 p$ we have

$$
\left[2 p(q+a p)-v_{e}-2 p\right] \cdot a+b=0
$$

or

$$
\left[2 p^{2}(a-\mathrm{I})-v_{e}\right] \cdot a+b=0 .
$$

But

and so

$$
v_{e}+v_{e}^{*}=2 p^{2}(\alpha-\mathrm{r})
$$

$$
a=b /\left(-v_{e}^{*}\right) \text {. }
$$

Similarly, for the latent root $\lambda=v_{e}^{*}+2 p$,

$$
a=b /\left(-v_{e}\right) \text {. }
$$


Thus if the principal components are $\mathrm{A}$ and $\mathrm{B}$, we have

$$
\left(\begin{array}{l}
A \\
B
\end{array}\right)=\left(\begin{array}{l}
I-v_{e}^{*} \\
I-v_{e}
\end{array}\right) \cdot\left(\begin{array}{l}
y \\
x
\end{array}\right)
$$

or

$$
\left(\begin{array}{l}
y \\
x
\end{array}\right)=\left(\begin{array}{ll}
-v_{e} & v_{c}^{*} \\
-\mathrm{I} & \mathrm{I}
\end{array}\right) \cdot\left(\begin{array}{l}
\mathrm{A} \\
\mathrm{B}
\end{array}\right)
$$

where we are ignoring the divisor $\left|\begin{array}{l}\mathrm{I}-v_{e}^{*} \\ \mathrm{I}-v_{e}\end{array}\right|$ which will disappear when we put $v=y / x$.

Hence

and so

$$
\left(\begin{array}{l}
y_{n} \\
x_{n}
\end{array}\right)=\left(\begin{array}{cc}
-v_{e} & v_{e}^{*} \\
-\mathrm{I} & \mathrm{I}
\end{array}\right)\left(\begin{array}{c}
\left(v_{e}+2 p\right)^{n} y_{0}-v_{e}^{*}\left(v_{e}+2 p\right)^{n} x_{0} \\
\left(v_{e}^{*}+2 p\right)^{n} y_{0}-v_{e}\left(v_{e}^{*}+2 p\right)^{n} x_{0}
\end{array}\right)
$$

$$
v_{n}=\frac{v_{e}^{*}\left[\left(v_{e}^{*}+2 p\right) /\left(v_{e}+2 p\right)\right]^{n}\left(v_{0}-v_{e}\right)-v_{e}\left(v_{0}-v_{e}^{*}\right)}{\left[\left(v_{e}^{*}+2 p\right) /\left(v_{e}+2 p\right)\right]^{n}\left(v_{0}-v_{e}\right)-\left(v_{0}-v_{e}^{*}\right)} .
$$

At $n=\infty$ we must have $v_{\infty}=v_{e}$, or $\left(v_{e}^{*}+2 p\right) /\left(v_{e}+2 p\right)<\mathrm{I}$; that is $v_{e}^{*}<v_{e}$ or $(1-\alpha)\left[p^{2}(1-\alpha)+4 q\right]>0$. Thus the stability condition is simply $a<\mathrm{I}$ which is the case unless mating is completely assortive. It is impossible in the present context to have $\alpha>\mathrm{I}$; in any mathematically equivalent system in which this were possible, it would imply a stable equilibrium at $v_{c}^{*}$.

Mating completely assortive (Case of $\alpha=I$ )

When $\alpha=\mathrm{I}$, we have $v_{e}=v_{e}^{*}=0$ and

$$
v_{n}=\frac{v_{e}^{*}\left(v_{0}-v_{e}\right)-v_{e}\left(v_{0}-v_{e}^{*}\right)}{\left(v_{0}-v_{e}\right)-\left(v_{0}-v_{e}^{*}\right)}=0 / 0
$$

and cannot thus be determined from this equation. If we return to the original matrix, however, which when $\alpha=$ I becomes

$$
\left(\begin{array}{cc}
2 p & 0 \\
1 & 2 p
\end{array}\right)
$$

then direct multiplication suggests

$$
\left(\begin{array}{cc}
2 p & 0 \\
1 & 2 p
\end{array}\right)^{n}=\left(\begin{array}{cc}
(2 p)^{n} & 0 \\
n(2 p)^{n-1} & (2 p)^{n}
\end{array}\right)
$$

which is easily proved by induction.

Hence

which becomes simply

$$
v_{n}=\frac{(2 p)^{n} v_{0}}{n(2 p)^{n-1} v_{0}+(2 p)^{n}}
$$

$$
v_{n}=2 p v_{0} /\left(n v_{0}+2 p\right)
$$


Thus in the simplest case of $v_{0}=\frac{1}{2}$ and $p=\frac{1}{2}$, we find $v_{n}=1 /(n+2)$, giving $v_{1}=\frac{1}{3}, v_{2}=\frac{1}{4}, v_{3}=\frac{1}{5}$, etc., a result obtained by Jennings (1916).

Table I shows the approach to equilibrium for five different values of $a$ when gene frequencies are equal. Wright (1921) has given similar values for $a=0.8$ and 0.5 , though he does not develop any general formulæ.

TABLE I

\begin{tabular}{|c|l|l|l|l|l|}
\hline \multicolumn{1}{|c|}{$\alpha=$} & 0.1 & 0.3 & 0.5 & 0.7 & 0.9 \\
\hline$n=0$ & 0.5 & 0.5 & 0.5 & 0.5 & 0.5 \\
\hline 1 & 0.4833 & 0.4500 & 0.4167 & 0.3833 & 0.35 \\
2 & 0.4826 & 0.4431 & 0.3971 & 0.340 & 0.2833 \\
3 & 0.4825 & 0.4421 & 0.3921 & 0.3292 & 0.2487 \\
5 & 0.4825 & 0.4420 & 0.3905 & 0.3210 & 0.2178 \\
8 & 0.4825 & 0.4419 & 0.3904 & 0.3196 & 0.2042 \\
13 & 0.4825 & 0.449 & 0.3904 & 0.3195 & 0.2004 \\
$\infty$ & 0.4825 & 0.4419 & 0.3904 & 0.3195 & 0.2 \\
\hline
\end{tabular}

Thus equilibrium for small values of $a$ lies rather close to that for random mating, and in all cases equilibrium is rapidly attained unless $a$ is almost unity.

\section{ASSORTIVE MATING WITHOUT COMPLETE DOMINANCE}

The system now to be examined is that in which each genotype has a separate probability of mating with a like genotype. As before, let the genotypic proportions of $\mathrm{AA}, \mathrm{AB}$ and $\mathrm{BB}$ be $u, v$ and $w$, and let $\alpha, \beta$ and $\gamma$ be the respective probabilities of assortive mating. It will then be assumed that the proportion $\mathrm{R}=\mathrm{I}-(\alpha u+\beta v+\gamma w)$ of the population mates at random. The frequencies of the mating types will be as follows.

\begin{tabular}{cl} 
Mating type & \multicolumn{1}{c}{ Frequency } \\
$\mathrm{AA} \times \mathrm{AA}$ & $\alpha u+(\mathrm{I}-\alpha)^{2} u^{2} / \mathrm{R}$ \\
$\mathrm{AA} \times \mathrm{AB}$ & $2(\mathrm{I}-\alpha)(\mathrm{I}-\beta) u v / \mathrm{R}$ \\
$\mathrm{AA} \times \mathrm{BB}$ & $2(\mathrm{I}-\alpha)(\mathrm{I}-\gamma) u w / \mathrm{R}$ \\
$\mathrm{AB} \times \mathrm{AB}$ & $\beta v+(\mathrm{I}-\beta)^{2} v^{2} / \mathrm{R}$ \\
$\mathrm{AB} \times \mathrm{BB}$ & $2(\mathrm{I}-\beta)(\mathrm{I}-\gamma) v w / \mathrm{R}$ \\
$\mathrm{BB} \times \mathrm{BB}$ & $\gamma w+(\mathrm{I}-\gamma)^{2} w^{2} / \mathrm{R}$
\end{tabular}

Thus if $u^{\prime}, v^{\prime}$ and $w^{\prime}$ represent the genotypic proportions in the next generation, we have

$$
\begin{aligned}
u^{\prime} & =\alpha u+\frac{1}{4} \beta v+\left[(\mathrm{I}-\alpha) u+\frac{1}{2}(\mathrm{I}-\beta) v\right]^{2} / \mathrm{R} \\
v^{\prime} & =\frac{1}{2} \beta v+2\left[(\mathrm{I}-\alpha) u+\frac{1}{2}(\mathrm{I}-\beta) v\right] \times\left[(\mathrm{I}-\gamma) w+\frac{1}{2}(\mathrm{I}-\beta) v\right] / \mathrm{R} \\
w^{\prime} & =\gamma w+\frac{1}{4} \beta v+\left[(\mathrm{I}-\gamma) w+\frac{1}{2}(\mathrm{I}-\beta) v\right]^{2} / \mathrm{R} .
\end{aligned}
$$

It may easily be shown that $u^{\prime}+\frac{1}{2} v^{\prime}=u+\frac{1}{2} v$ and hence that the gene frequency remains constant. Thus we may put $u=p-\frac{1}{2} v$ and $w=q-\frac{1}{2} v$, 
where $p$ and $q$ are the frequencies of the alleles $\mathrm{A}$ and $\mathrm{B}$ respectively. Substitution leads, after some reduction, to a recurrence relation in $v$ alone:

$$
v^{\prime}=\frac{1}{2} \beta v+\frac{2\left[(\mathrm{I}-\alpha) p+\frac{1}{2}(\alpha-\beta) v\right]\left[(\mathrm{I}-\gamma) q+\frac{1}{2}(\gamma-\beta) v\right] .}{\mathrm{I}-(\alpha p+\gamma q)+v\left(\frac{1}{2} \alpha-\beta+\frac{1}{2} \gamma\right)} .
$$

In the simple case of $\alpha=\beta=\gamma$, the recurrence relation reduces to that for mixed selfing and random mating:

$$
v^{\prime}=\frac{1}{2} \alpha v+2 p q(\mathrm{I}-\alpha) .
$$

Thus at equilibrium $v_{e}=2 p q(\mathrm{I}-\alpha) /\left(\mathrm{I}-\frac{1}{2} \alpha\right)$. For stability we must have $\frac{1}{2} \alpha<\mathrm{I}$ or $\alpha<2$, and the rate of approach to equilibrium will be determined by $-\log _{e} \frac{1}{2} \alpha$. This model never reduces to that of complete dominance, however, since all genotypes are assumed to be recognisably distinct.

A linear equation may be obtained in the general case if it is assumed that $\alpha, \beta$ and $\gamma$ are small, so that quadratic and higher terms in these parameters may be ignored. This gives

$$
v^{\prime}=\left(2 p q \beta-\frac{1}{2} \beta+\alpha q^{2}+\gamma p^{2}\right) v+2 p q(\mathrm{I}-\alpha q-\gamma p),
$$

and at equilibrium

$$
v_{e}=2 p q\left(\mathrm{I}-\frac{1}{2} \beta+2 \beta p q-\alpha p q-\gamma p q\right) .
$$

For stability $2 \beta p q-\frac{1}{2} \beta+\alpha q^{2}+\gamma p^{2}<\mathrm{r}$; when $p=q$ this condition becomes $\frac{1}{4} \alpha+\frac{1}{4} \gamma<$ I. If $\alpha=\gamma$ this reduces to the stability condition found exactly for the equilibrium between mixed selfing and random mating. In general, whenever the parameters $\alpha, \beta$ and $\gamma$ are at all small, this solution will be very accurate. Thus when $\alpha=\gamma=\frac{1}{2}$ and $p=q, v_{e}=\mathrm{I} / 3$ exactly, but $3 / 8$ by the approximate treatment. This exceeds the exact value by $\mathrm{I} / 24$. If, however, $\alpha=\gamma=\frac{1}{4}$ and $p=q$, the approximate value of $v_{e}$ exceeds the exact value of $3 / 7$ by only $\mathrm{I} / \mathrm{I}$ I 2 . An exact solution of the general recurrence equation, however, is available as one of the roots of the quadratic

$$
\begin{gathered}
{\left[\left(\mathrm{I}-\frac{1}{2} \beta\right)\left(\frac{1}{2} \alpha-\beta+\frac{1}{2} \gamma\right)-\frac{1}{2}(\alpha-\beta)(\gamma-\beta)\right] v^{2}+\left[\left(\mathrm{I}-\frac{1}{2} \beta\right)(\mathrm{I}-\alpha p-\gamma q)\right.} \\
-(\mathrm{I}-\gamma)(\alpha-\beta) q-(\mathrm{I}-\alpha)(\gamma-\beta) p] v-2 p q(\mathrm{I}-\alpha)(\mathrm{I}-\gamma)=0 .
\end{gathered}
$$

Thus if $\alpha=0.3, \beta=0.2$ and $\gamma=0.1$ and $p=q, v_{e}=0.4423$, whereas the approximate formula gives $0 \cdot 45$. When $\alpha, \beta$ and $\gamma$ become very small, however, the exact formula becomes very troublesome owing to the need to extract a square root to many places of decimals. As an example, when $\alpha=0.003, \beta=0.002$ and $\gamma=0.001$,

$$
v_{e}=\frac{-1 \cdot 994002+\sqrt{3 \cdot 976047960016}}{0 \cdot 000002} .
$$


The approximate method gives $0 \cdot 4995$, while to obtain this accuracy from the exact treatment, the above square root has to be extracted to ten significant figures as I 994002999 . In this case $-\log _{e}\left(\frac{1}{4} \alpha+\frac{1}{4} \gamma\right)$ $=6.907755$. For complete selfing, with no random mating, the equivalent value is $0.693 \mathrm{I} 5$. Thus equilibrium is approached 9.966 times as fast as homozygosity is approached in complete selfing.

\section{SUMMARY}

The object of this paper has been to discuss the origin of assortive mating and its effects on populations in which two alleles are segregating. The calculations given in the last two sections demonstrate that in a population in which a few individuals mate only with like mates, the equilibrium position, which is rapidly approached, lies rather close to that of random mating. Thus in a population that is in the process of splitting into two groups, assortive mating, if only slightly developed, should be a relatively small factor in the genetic isolation of the diverging groups. Darwinian selection, however, can operate only by the differential survival of individuals, and in the relative numbers of offspring they produce. From this point of view it is clear that the evolution of assortive mating will, in general, be rather slow. Only if in some individuals the preference for assortive mating is strongly developed will the selective forces discussed in Section 3 have large effects on which to act.

Acknowledgment.-This work was done during the tenure of a Nature Conservancy Research Studentship.

\section{REFERENCES}

COOCH, F. G., AND BEARDMORE, J. A. I959. Assortive mating and reciprocal difference in the Blue-Snow Goose complex. Nature, 183 , I833-1 834 .

FISHER, R. A. I9I8. The correlation between relatives on the supposition of Mendelian inheritance. Trans. Roy. Soc. Edin., 52, 399-433.

Fisher, R. A. 1930. The Genetical Theory of Natural Selection. Clarendon Press, Oxford.

FISHER, R. A. I949. The Theory of Inbreeding. Oliver and Boyd, Edinburgh.

JENnINGS, H. S. I9I6. The numerical results of diverse systems of breeding. Genetics, $I, 53-89$.

MATHER, K. I955. Polymorphism as an outcome of disruptive selection. Evolution, $9,52-6 \mathrm{I}$.

O'Donald, P. I959. Possibility of assortive mating in the Arctic Skua. Nature, 183, I 2 IO-I 2 I I.

WRIGHT, s. I92I. Systems of mating. III. Assortive mating based on somatic resemblance. Genetics, 6, I43-16r. 